TREATMENT OF UNCOMPLICATED URINARY TRACT

19 INFECTIONS WITH VARIOUS DOSES OF AMOXICILLIN. Abdollah Iravani, George A. Richard (Spon. by J.A. Mangos), University of Florida, College of Medicine, Department of Pediatrics, Gainesville.

On 182 occasions college coeds with acute, uncomplicated (UTIS), documented by $>10^{5}$ of the same bacteria in two consecutive urine cultures, were randomly treated with amoxicillin $3 \mathrm{gm}$., single dose (83); $3 \mathrm{gm}$. double dose at 12 hour intervals (70); and $250 \mathrm{mg}$. t.i.d. for 10 days (29). Clinical cure rates at one week post therapy in single dose group was $88.0 \%$ double dose group $80.0 \%$, and in 10 day group, $100 \%$. At four weeks post therapy in the single dose group, bacteriological cure rate was $68.7 \%$, reinfections $19.3 \%$, relapse $4.8 \%$ and persistence of infection $7.2 \%$; in the double dose group, bacteriological cure rate was $71.4 \%$, reinfection $7.1 \%$, relapse $12.9 \%$, and persistence of infection $8.6 \%$; in 10 day group, bacteriological cure rate was $82.8 \%$, reinfection $3.8 \%$, relapse $3.4 \%$, and no persistence of infection.

Conventional Amoxicillin therapy appeared superior to single and double dose; however, the differences were not statistically significant. Single dose appeared easy, safe and practical in acute, uncomplicated UTIs of young women. The drug was tolerated well in all pateints. Complete blood counts, blood chemistries and transaminase enzymes remained normal.
22 AGGRESSIVE BEHAVIORS IN YOUNG MOTHERS: MARKERS OF FUTURE MORBIDITY? Ruth A. Lawrence, Elizabeth R. McAnarney, Marilyn J. Aten, Howard P. Iker, Clara P. Baldwin and Alfred L. Baldwin (Spon. by Gilbert B. Forbes) U. of Roch. School of Med., Strong Mem. Hosp., Dept. of Peds., Roch., NY On the basis of clinical observations we became aware of aggressive behavior among adolescent mothers directed to their newborn infants characterized primarily by picking, poking, \& pinching. A study was designed to quantitate these behaviors. Ten minute individual non-feeding mother-infant interaction sequences for 34 adolescent mothers under nineteen years of age and 12 adult mothers were videotaped in a standard setting during the first three postpartum days. The tapes were scored by two observers who counted the frequency of behaviors observed over ten minutes. Intercoder reliability was greater than 0.90 on all behaviors scored. Videotapes of 46 subjects were randomly ordered \& raters were blind to the study hypothesis and maternal age. Twelve (12) of thirty-four (34) adolescents and one (1) of twelve (12) adults were observed to have some aggressive behaviors. When the number of adolescents observed in aggressive behaviors is contrasted with expectancies based on observation of adult behaviors the difference is highly significant $(p<.001)$. A more general hypothesis associating age and aggressive behavior is marginally significant with adolescents showing more and adults less aggressive behavior than chance would dictate $(p<.0725)$. We suggest that these aggressive behaviors seen predominantly among adolescent mothers may be used as markers of future social morbidity such as inadequate mothering of their infants ard children.
URINARY TRACT INFECTION CAUSED BY

20 STAPHYLOCOCCUS SAPROPHYTICUS (SS). by J.A. Mangos), University of Florida, College of Medicine, Department of Pediatrics, Gainesville.

In 492 college coeds, with acute, uncomplicated UTIs, documented by $>10^{5}$ colonies per $\mathrm{ml}$. of the same bacteria in 2 or 3 consecutive urine cultures, E. coli and novobiocin resistant (SS) comprised $76.0 \%$ and other bacteria $11.0 \%$. The in-vitro sensitivity testing revealed that SS were $100 \%$ sensitive to trimethoprim, trimethoprim/sulfamethoxozole, nitrofurantoin, and cephalexin; $91.0 \%$ to ampicillin; $93.0 \%$ to sulfasoxozole and $0.0 \%$ to nalidixic acid.

Patients with either E. coli or SS organism presented with similar symptoms of an acute lower tract infection. Costovertebral angle tenderness was noted in $21.0 \%$ of the patients with SS and in $10.0 \%$ of those with E. coli. Fever $>99.5 \mathrm{C}$. was present in $15.0 \%$ of patients with SS and in $25.0 \%$ of those with E. coli. Clinical response to therapy was similar for both groups. Isolation of SS from the periurethral (52.0\%), vaginal (31.0\%), and rectal (11.5\%) areas could only be accomplished in patients who had this organism as the primary urinary pathogen. SS was the second most common urinary pathogen isolated after $\mathrm{E}$. coli in young women.

\section{- 21}

A STUDY OF THE ASSOCIATION BETWEEN NEURODEVELOPMENTAL DEFICITS AND JUVENILE DELINQUENCX. Wa1ter Karniski, Melvin D. Levine, SImon Clarke, Judith Palfrey. Children's Hospital Medical Center, Division of Ambulatory Pediatrics, Boston, Massachusetts.

In young children, there has been documentation of an assoclation between neurodevelopmental dysfunctions and problems with learning, behavior, and soctal adjustment. Less is known about this association in adolescents. An adolescent neurodevelopmental examination was compiled and administered to 54 delinquents and 51 secondary school students. Six areas were assessed. There was no significant differences in the prevalence of minor neurological signs $(p=.37)$ or in gross motor function $(p=.02)$. The delinquents showed more deficits of fine motor function $(p=.02)$ and sequential memory $(p=.04)$. The greatest differences were in visual processing $(p=.002)$ and auditory-1.anguage function ( $p=.001)$. E1ghteen percent of delinquents and $4 \%$ of the comparison group were defictent in 2 or more neurodevelopmental areas. Nine percent of the delinquents and none of the comparison group had 3 or more dysfunctions.

There is a high prevalence of chronic developmental dysfunction among delinquent adolescents. These may have been undiagnosed, poorly managed, or resistant to treatment.

A neurodevelopmental examination is a useful diagnostic tool for identifying endogenous factors in behavior, learning, and adjustment problems of this age group. Such findings have implications for policy and formulation of individualized management strategies.
ACCEPTANCE OF MULTIPLE INSULIN INJECTIONS BY ADOLESCENT DIABETICS. Doris H. Milman, Salvador Castells, Marie-Silmarilita Noval. Dept. of Ped., Downstate Medica1 Center, SUNY, Brooklyn, New York

Nine adolescent diabetics, 6 males and 3 females from 19 yr $10 \mathrm{~m}$ to $13 \mathrm{yr} 1 \mathrm{~m}$ on poor diabetic control on one injection of NPH and Regular insulins daily, were placed on good diabetic control with two injections of NPH and Regular insulins daily. Good diabetic control was def ined by fasting blood sugars below $200 \mathrm{mg}$ per $100 \mathrm{ml}$ and plasma levels of $\mathrm{HgA}_{1 \mathrm{C}}$ within one SD below the normal mean for 12 months. At the time of their hospitalization for stabilization on the new treatment regimen, patients emotional adjustment was evaluated by means of a structured interview plus figure drawings and data from parental and school sources. Acceptance of the new treatment regimen was positive in 8 of the 9 patients. There was only one instance of poor acceptance, a $13 \mathrm{yr}$ old boy, with a prior history of poor compliance. In the other 8 , there was a generally good prior adjustment to being diabetic. It was concluded that a more demanding treatment regimen did not add to adjustment problems in those adolescents who had previously achieved an emotional accommodation to their disease. ADOLESCENT POLYCYSTIC OVARY SYNDROME--OUT-
24 PATIENT DIAGNOSIS. George W. Moll, Jr.,* Robert L.
Rosenfield, and Barry H. Rich, * Univ. of Chicago Pritzker

School of Med., Dept

We have expanded the endocrine diagnostic criteria for PCOS and have identified it in 8 adolescents. PCOS consists of a spectrum of functional ovarian hyperandrogenic states ranging from cases without histologic ovarian abnormalities through classic Stein-Leventhal ovaries. Diagnostic criteria developed for adults are: elevated plasma free (unbound) testosterone $(\mathrm{T})(>12 \mathrm{pg} / \mathrm{ml})$ which does not fall to $\leq 8$ upon adequate adrenal suppression by dexamethasone (dex). In addition, we found bioactive $L H(B-L H)$ to be more of ten significantly elevated in PCOS than radioimmunoassayable $\mathrm{LH}(\mathrm{I}-\mathrm{LH})$.

We first established that adult female levels of total and free $T$ and LH are normally achieved prior to the time of menarche.

The 8 PCOS patients were 12.5-17 years old. 5 had menstrual disturbances ( 4 oligo- or amenorrhea $\times 1.5-2.5$ yrs and 1 menometrorrhagia $\times 1.5 \mathrm{yrs}), 6$ had hirsutism, and 5 were obese. 1 with subclinical problems was detected in a school screening program. A high free $T$ level was found in each $(15-25 \mathrm{pg} / \mathrm{ml})$, though total $\mathrm{T}(51-133 \mathrm{ng} / \mathrm{dl})$ and free $T$ (10.1-25) were intermittently normal in some. Free $T$ fell only to 9-28 $\mathrm{pg} / \mathrm{ml}$ on dex. B-LH was elevated in $4 / 6(>620 \mathrm{ng} / \mathrm{ml})$, while $\mathrm{I}-\mathrm{LH}$ was only elevated in $2 / 6(>91 \mathrm{ng} / \mathrm{ml})$. I patient with persistently high I-LH $(260-410 \mathrm{ng} / \mathrm{ml})$ underwent laparoscopy which revealed sclerocystic ovaries. Estrogen-progestin $(B C P)(n=3)$ normalized free $T(\leq 8 \mathrm{pg} / \mathrm{ml})$.

PCOS, as the basis of teenage menstrual difficulties or hirsutismsometimes minor, can be diagnosed from elevated free $T$ levels pre and post dex and elevated B-LH without invasive procedures. 